\section{Analytical Comparison of Two Multiscale Coupling Methods for Nonlinear Solid Mechanics}

Daria Koliesnikova

CEA, DES, IRESNE, DEC, SESC, F-13108 Saint-Paul-lez-Durance, France;

Aix-Marseille Université, CNRS, Centrale Marseille, LMA, 4 Impasse Nikola Tesla, CS 40006,

F-13453 Marseille Cedex 13, France

e-mail: daria.koliesnikova@cea.fr

\section{Isabelle Ramière}

CEA, DES, IRESNE, DEC, SESC,

F-13108 Saint-Paul-lez-Durance, France

e-mail: isabelle.ramiere@cea.fr

\section{Frédéric Lebon}

Aix-Marseille Université, CNRS, Centrale Marseille, LMA, 4 Impasse Nikola Tesla, CS 40006,

F-13453 Marseille Cedex 13, France

e-mail: lebon@lma.cnrs-mrs.fr

The aim of this work is to compare two existing multilevel computational approaches coming from two different families of multiscale methods in a nonlinear solid mechanics framework. A locally adaptive multigrid method and a numerical homogenization technique are considered. Both classes of methods aim to enrich a global model representing the structure's behavior with more sophisticated local models depicting fine localized phenomena. It is clearly shown that even being developed with different vocations, such approaches reveal several common features. The main conceptual difference relying on the scale separation condition has finally a limited influence on the algorithmic aspects. Hence, this comparison enables to highlight a unified framework for multiscale coupling methods.

Keywords: multiscale, coupling, solid mechanics, local multigrid, numerical homogenization, $L D C, F E^{2}$

\section{Introduction}

In many engineering situations, it is crucial to understand and precisely model certain complex phenomena with the necessity to capture multiple length scales features. A complete resolution at the finest details scale may rapidly become prohibitive in terms of computational complexity. As a remedy to such issue, a common approach in practical engineering analysis is to rely on multiscale coupling methods permitting to reach a desired precision locally while avoiding the necessity to solve a complete fine-scale problem.

The choice of the computational strategy to be applied is governed by the length scale ratio $(l / L)$ between the characteristic size of the local effects $(l)$ and of the structural level $(L)$. Generally, two classes of multiscale problems can be distinguished [1]. The first class incorporates problems with the small-scale difference $(l / L \approx 1 / 4, \ldots, 1 / 10)$, cf. Fig. 1(a). Local phenomena, such as local concentrations or singularities, discontinuous boundary conditions, cracks, etc., belong to the macroscopic or mesoscopic scale. To deal with this kind of problems, adaptive mesh refinement techniques
[2-4] are generally well suited. For the second class of problems, the scale difference is much more pronounced $(l / L \ll 1)$ : fine phenomena are localized at the microscopic scale (heterogeneous media, composite materials, etc.), cf. Fig. 1(b). For such problems, analytical [5,6] and numerical [7-9] homogenization approaches have been developed.

We focus here on multilevel approaches lying on addressing a problem from different levels of details, coupled in the same computational scheme. Commonly, a global model aims to capture a structure's global behavior, while local model offers more accurate simulation of fine phenomena. The objective of this study is to place in the same unified multilevel framework two existing multiscale methods, respectively, from each of both previously mentioned classes. For the first class of multiscale methods, locally adaptive multigrid methods are considered here, as they are efficient multilevel mesh refinement techniques [10-12]. We focus on the local defect correction (LDC) method [11] as it seems to be the most pertinent choice for solid mechanics problems [13]. For the second class of multiscale methods, numerical homogenization approaches are chosen. Among them, the so-called $\mathrm{FE}^{2}$ (finite element square) approach $[8,14]$ is considered here as it is the numerical reference, especially when analytical methods (based for example on HashinShtrikman [15] or Mori-Tanaka [16] models) find their limits (particular geometries, large number of material phases, complex behavior,...).

It has to be underlined that the scope of this work is not to develop a new strategy of using efficient multigrid solvers in a homogenization context as made in Refs. [17-19], but to analytically compare two existing multilevel methods coming from different multiscale classes.

\section{Nonlinear Quasi-Static Problem}

In this work, we place ourselves in a general fully nonlinear framework. A mechanical quasi-static nonlinear boundary-value problem of the following form is considered:

$$
\begin{cases}\operatorname{div} \boldsymbol{\sigma}=0 & \text { in } \Omega \\ \boldsymbol{\varepsilon}(\mathbf{u})=\frac{1}{2}\left(\operatorname{grad} \mathbf{u}+\operatorname{grad}^{\mathrm{T}} \mathbf{u}\right) & \text { in } \Omega \\ \boldsymbol{\sigma}=\mathcal{F}(\boldsymbol{\varepsilon}, \dot{\boldsymbol{\varepsilon}}, \ldots) & \text { in } \Omega \\ \mathbf{u}=\mathbf{u}_{D} & \text { on } \Gamma_{\mathbf{D}} \\ \boldsymbol{\sigma} \cdot \mathbf{n}=\mathbf{F}_{\mathbf{N}} & \text { on } \Gamma_{\mathbf{N}}\end{cases}
$$

with $\Omega$ being an open bounded subset of $\mathbb{R}^{D}$ — the domain occupied by the solid, with $D$ referring to the problem's dimension. The boundary $\Gamma$ of $\Omega$ is partitioned into $\Gamma_{\mathbf{D}}$ (Dirichlet boundary conditions) and $\Gamma_{\mathbf{N}}$ (Neumann boundary conditions), with $\Gamma_{\mathbf{D}} \cup$ $\Gamma_{\mathbf{N}}=\partial \Omega$ and $\Gamma_{\mathbf{D}} \cap \Gamma_{\mathbf{N}}=\varnothing$.

The constitutive law relating strain and stress tensors is given by the third equation of Eq. (1). This relation is considered nonlinear; hence, the problem (1) is nonlinear in terms of strain and therefore in terms of displacement. Generally, a Newton-like algorithm is applied to linearize the variational formulation of the problem (1). The following variational form is then obtained at iteration $k$ :

$$
\begin{aligned}
\int_{\Omega} \boldsymbol{\varepsilon}(\mathbf{v}): \mathbf{H}^{k-1}: \boldsymbol{\varepsilon}\left(\mathbf{u}^{k}-\mathbf{u}^{k-1}\right) \mathrm{d} \Omega \\
\quad=\int_{\Gamma_{\mathbf{N}}} \mathbf{F}_{N} \mathbf{v} \mathrm{d} \Gamma_{\mathbf{N}}-\int_{\Omega} \boldsymbol{\varepsilon}(\mathbf{v}): \boldsymbol{\sigma}^{k-1} \mathrm{~d} \Omega
\end{aligned}
$$

for all $\mathbf{v}$ kinematically admissible to zero and $\mathbf{H}$ being a linearization operator. In the classical Newton method, $\mathbf{H}^{k-1}$ is chosen to be the Jacobian matrix - the so-called constitutive tangent matrix in solid mechanics. In quasi-Newton scheme, this tangent matrix is generally approximated by a more easily computable matrix (secant matrix, constant matrix, etc.). The choice of the operator $\mathbf{H}$ only affects the convergence order of the obtained algorithm and may not impact the final accuracy. 

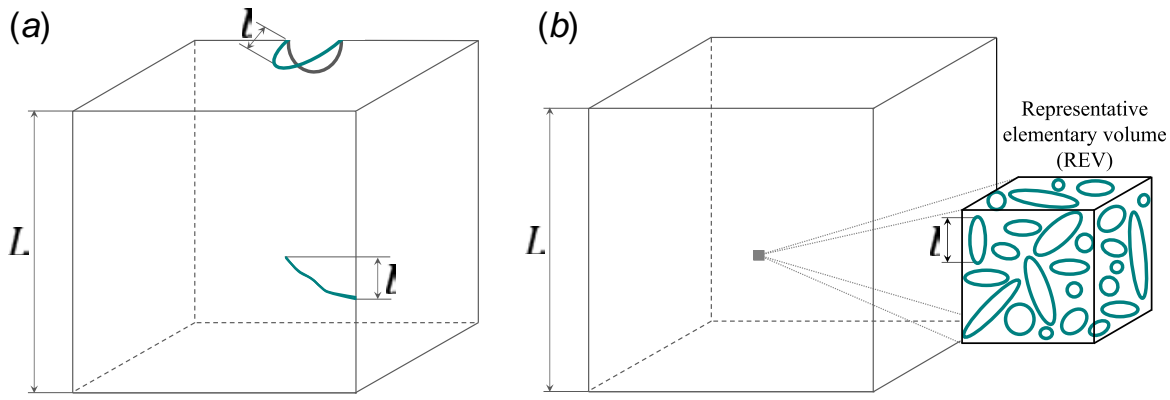

Fig. 1 Two classes of multiscale problems: problems involving effects localized (a) at the macroscopic scale $(I \leq L)$ and $(b)$ at the microscopic scale $(I \ll L)$

The iterative Newton-like algorithm tends to minimize the residual $R^{k-1}=\int_{\Gamma_{\mathbf{N}}} \mathbf{F}_{N} \mathbf{v} \mathrm{d} \Gamma_{\mathbf{N}}-\int_{\Omega} \boldsymbol{\varepsilon}(\mathbf{v}): \boldsymbol{\sigma}^{k-1} \mathrm{~d} \Omega$, where $\boldsymbol{\sigma}^{k-1}$ is updated using the nonlinear constitutive relation at each iteration. For the sake of simplicity, we consider here a rate-independent constitutive relation of the following form:

$$
\boldsymbol{\sigma}^{k}=\mathcal{F}\left(\boldsymbol{\varepsilon}\left(\mathbf{u}^{k}\right)\right)
$$

We assume that the considered problem is solved using the standard finite element method in displacement. By denoting [-], the discretized quantities, and $[K]$ the stiffness matrix, the problem (2) can be written as

$$
\left[K^{k-1}\right]\left[\delta U^{k}\right]=\left[F^{e x t}\right]-\left[F^{i n t, k-1}\left(\boldsymbol{\sigma}^{k-1}\right)\right]
$$

with $\left[R^{k-1}\right]=\left[F^{e x t}\right]-\left[F^{i n t, k-1}\left(\boldsymbol{\sigma}^{k-1}\right)\right]$ and $\left[\delta U^{k}\right]=\left[U^{k}\right]-\left[U^{k-1}\right]$. Numerically speaking, integration of the constitutive relation (3) is only performed at integration points. The algorithm of the standard resolution process is summarized in Algorithm 1.

Algorithm 1 Finite element resolution algorithm for nonlinear quasi-static problem

for $k=1$ until convergence do

- Solve the equilibrium equation (4)

- Integrate the constitutive relation (3) at each integration point to obtain $\boldsymbol{\sigma}^{k}$

- Update the residual: $\left[R^{k}\right]=\left[F^{\text {ext }}\right]-\left[F^{\text {int }, k}\left(\boldsymbol{\sigma}^{k}\right)\right]$

- Check the convergence on $\left[R^{k}\right]$

end

\section{Hybrid $\mathrm{FE}^{2}$ and Local Defect Correction Multilevel Algorithms}

In what follows, we aim to rewrite both (see Sec. 1) multilevel algorithms in a unified setting. For this study, we limit ourselves to two levels (referred to as global and local); however, it is straightforward for both considered multiscale methods to increase the number of levels. On the global computational domain, named $\Omega_{M}$, a global homogenized model is defined. We assume that in some region $\hat{\Omega}_{M} \subset \Omega_{M}$, local mesh $\Omega_{m}$ aiming to solve local nonlinear problem(s) is added. The quantities derived on the global level are marked as $\cdot_{M}$, while those associated with the local level as $\cdot_{m}$. The two-level iterative nonlinear resolution derived from both methods can be represented in general common way, see Fig. 2.

3.1 Adaptive Multigrid Local Defect Correction Method. Adaptive local multigrid methods have been initially introduced in a multilevel mesh refinement context. These techniques consist in improving the solution's accuracy locally near critical regions (local concentrations or singularities, crack tip, re-entrant corner, etc.) by adding local levels with finer meshes. Thus, they exploit

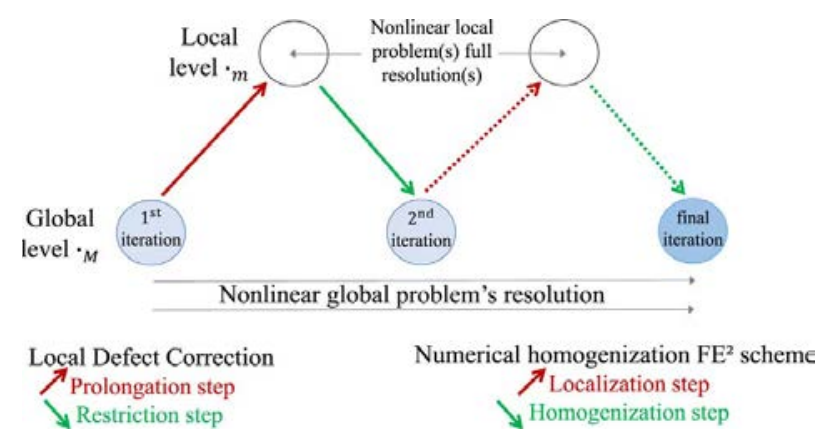

Fig. 2 Generic two-level iterative process for nonlinear problems multiscale resolution

the inverse hierarchy of grids compared with standard multigrid methods [20]. The inter-grid transfer operators are used to link the generated levels of grids.

These adaptive multigrid approaches can also be easily exploited in a multimodel context. It consists in adding appropriate local models in critical zones, where the global model is no more representative. These local models aim to precisely capture local features, such as geometrical nonlinearities [21], local plasticity regions [22], cracks [23], etc. In Ref. [24], the authors proposed to adapt the local multigrid methods to treat homogenization problems via the use of appropriate homogenization-based transfer operators. These operators are supposed to ensure the coincidence of the restricted local stiffness matrix with the homogenized global one.

We consider in this study the Local Defect Correction approach [11] with a homogenized model defined on the global level and an adapted fine model on the local level. This local level recovers the critical part of the global domain $\left(\Omega_{m}=\hat{\Omega}_{M}\right)$, see Fig. 3(a).

In the nonlinear context, there exist two possible ways to carry out the two embedded iterative processes (nonlinear problem's resolution and LDC multigrid resolution). In this paper, we focus on a nonlinear LDC iterative process performed with only one smoothing operation done at the global level, while on the local level, the nonlinear problem's resolution is pushed until convergence, cf. Fig. 2. It allows to benefit from the nonlinear local problem's resolution to correct the global problem within Newton iterations. We assume the computations at each generated level to be performed using the standard finite element method. Hence, we introduce in Algorithm 2, a two-scale LDC method for nonlinear solid mechanics. To the best of our knowledge, only Ref. [25] has provided an application of LDC method for nonlinear solid mechanics. It is the first time here that LDC algorithm is extended in a solid mechanics multimodel context.

The underlying idea of the LDC method is to make the global displacement tend toward the restricted local one $\left(\mathbf{u}_{M}^{k} \rightarrow \tilde{\mathbf{u}}_{M}^{k}\right)$ in a weak sense via the residual on $\hat{\Omega}_{M}$. Indeed, the LDC approach aims to define, based on local computations, a so-called defect which is added to the global problem's right-hand side. For nonlinear solid 
(a)
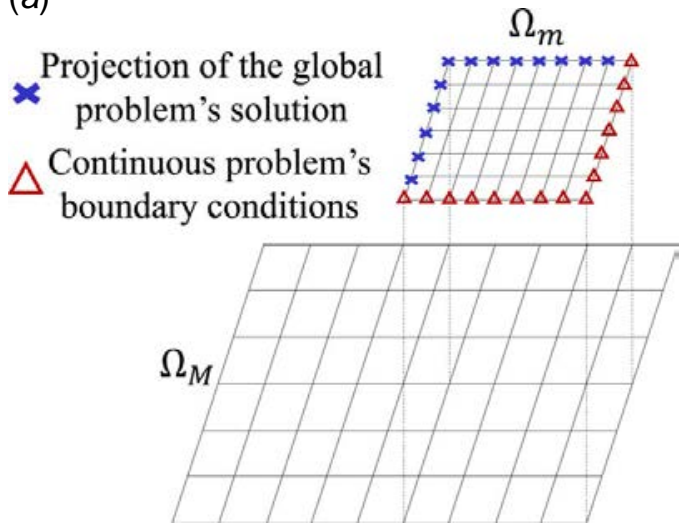

(b)

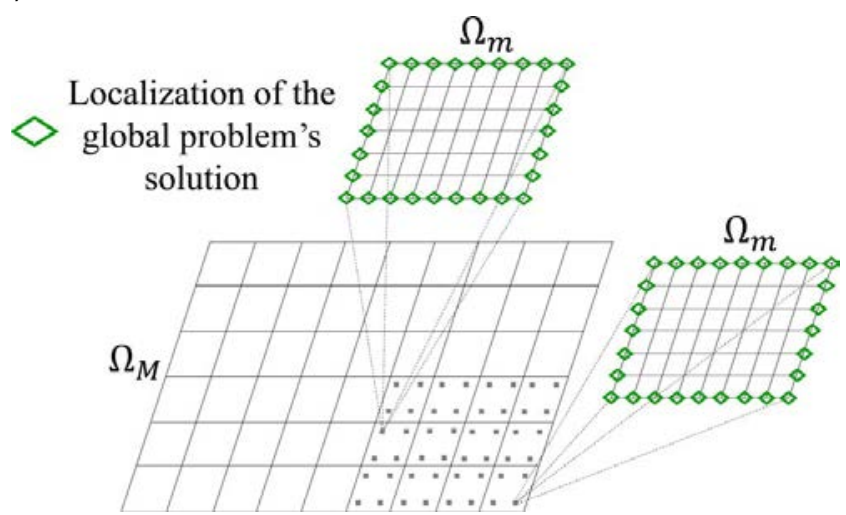

Fig. 3 Global and local levels with boundary conditions for the (a) LDC and (b) hybrid $\mathrm{FE}^{2}$ methods

mechanics problems, this defect is calculated in terms of a corrected stress associated to the restricted local displacement (Eq. (7)).

Finally, the algorithm returns to a minimization, in the region of interest $\Omega_{M}$, of the difference between the internal forces computed, respectively, via $\tilde{\boldsymbol{\sigma}}_{M}^{k}$ and via $\boldsymbol{\sigma}_{M}^{k}$. The internal forces to be equilibrated are now $\left[F_{M}^{i n t, k}\left(\tilde{\boldsymbol{\sigma}}_{M}^{k}\right)\right]$ which implicitly take into account the external forces via the local problem's boundary conditions. Note that this corrected stress $\tilde{\boldsymbol{\sigma}}_{M}^{k}$ has only an algorithmic meaning.

Algorithm 2 Two-scale Local Defect Correction algorithm for nonlinear quasi-static problem

for $k=1$ until convergence do

- Solve the global equilibrium equation (4) in $\Omega_{M}$

- In the region of interest $\hat{\Omega}_{M}$ : use the LDC model

- Global-to-local information transfer: prolongation step. Impose boundary conditions on the local mesh boundaries $\partial \Omega_{m}$ (cf. Fig. 3(a)): boundary conditions of the continuous problem on $\partial \boldsymbol{\Omega}_{m} \cap \partial \boldsymbol{\Omega}_{M}$ and imposed displacement derived by prolongating the global displacement on the fictive boundary $\Gamma_{f}:=\partial \Omega_{m} \backslash\left(\partial \Omega_{m} \cap \partial \Omega_{M}\right)$ :

$$
\boldsymbol{u}_{\Gamma_{f}}=\mathcal{P}_{M}^{m}\left(\boldsymbol{u}_{M}^{k}\right)
$$

with $\mathcal{P}_{M}^{m}$ a prolongation operator (typically interpolation operator)

○ Local problem: nonlinear problem's full resolution on $\Omega_{m}$ with Algorithm 1 using the local constitutive model

- Local-to-global information transfer: restriction step. Compute in $\hat{\Omega}_{M}$, the restricted local displacement:

$$
\tilde{\boldsymbol{u}}_{M}^{k}=\mathcal{R}_{m}^{M}\left(\boldsymbol{u}_{m}^{k}\right)
$$

with $\mathcal{R}_{m}^{M}\left(\neq\left(\mathcal{P}_{M}^{m}\right)^{T}\right)$ a restriction operator (interpolation or injection operator) and the associated global stress:

$$
\tilde{\boldsymbol{\sigma}}_{M}^{k}=\mathcal{F}_{M}\left(\boldsymbol{\varepsilon}\left(\tilde{\boldsymbol{u}}_{M}^{k}\right)\right)
$$

- In $\Omega_{M}$ : integration of the global constitutive law (Eq. (3)) to find $\sigma_{M}^{k}$ - Update the global residual:

$$
\left[R_{M}^{k}\right]= \begin{cases}{\left[F_{M}^{i n t, k}\left(\tilde{\boldsymbol{\sigma}}_{M}^{k}\right)\right]-\left[F_{M}^{i n t, k}\left(\boldsymbol{\sigma}_{M}^{k}\right)\right]} & \text { in } \hat{\Omega}_{M} \\ {\left[F_{M}^{e x t}\right]-\left[F_{M}^{i n t, k}\left(\boldsymbol{\sigma}_{M}^{k}\right)\right]} & \text { in } \Omega_{M} \backslash \hat{\Omega}_{M}\end{cases}
$$

- Check the convergence on $\left[R_{M}^{k}\right]$

end

3.2 Numerical Homogenization Hybrid $\mathrm{FE}^{2}$ Method. Historically, the homogenization methods have been widely used to treat the problems with the large-scale separation and thus have to verify two strong assumptions, cf. Refs. [1,5]. The first one states the scale separation in terms of dimensions $(l \ll L)$ which allows to consider the local averaged behavior to be representative of the global structure's behavior at a given point. The second hypothesis consists in assuming the homogeneity of the global strain near a macroscopic point of interest, which enables to prescribe the boundary conditions on the local level. This second hypothesis may limit the application of homogenization techniques in the presence of localized phenomena (crack, stress concentration, strong temperature gradient, etc.).

In contrast to the analytical homogenization methods $[5,15,16]$, where the microscopic behavior is obtained based on analytical formulations, the scope of the numerical homogenization approaches lies on the possibility to obtain the material's microstructural behavior on-the-fly by solving numerically the micro-scale problems. It permits to replace the integration of the global constitutive law by computations performed at the microscopic scale. It is of particular interest when the effective behavior is difficult to predict a priori. Local micro-scale problems are formulated on representative elementary volumes (REV) characteristic of the microstructure. These local domains are attached to macroscopic integration points, see Fig. 3(b). The scale transition is thus constructed on point-to-volume basis. Note, that the characteristic size of the REV is very small when compared with the size of the region of interest; moreover, one defines a unique discretization of the REV which is used for all local problems resolutions.

We consider in this study the numerical homogenization $\mathrm{FE}^{2}$ technique $[8,14]$ where the problems on both scales are recursively solved with the finite element method, cf. Fig. 2. Based on the homogenization theory, the $\mathrm{FE}^{2}$ technique directly defines the global stress as the average of the computed microscopic stress on the associated REV. The $\mathrm{FE}^{2}$ scheme permits to accurately compute on-the-fly the homogenized global stress as well as the complete local microstructural behavior. This approach can also be used to correct the global linearization operator $\mathbf{H}^{k-1}$ (via the effective tangent matrix obtained from the microscale computations, cf. for example, Refs. [26,27]). However, the evaluation of the effective tangent matrix requires to perform additional computations on the REV. This option, being computationally expensive, is not considered here. It results in limiting the convergence order of the Newton algorithm: the linear convergence is thus supposed.

In the case of real industrial applications, the numerical homogenization strategies, even being naturally parallelizable, are accompanied with a significant computational cost (large number of local micro-scale problems to be solved). Hence, we focus here on the hybrid $\mathrm{FE}^{2}$ technique [28] consisting in performing micro-scale computations only in region of interest, cf. Algorithm 3. It has to be noticed that in the literature complementary strategies aiming to reduce the microscale computational cost exist. Let us mention for example the multigrid preconditioning techniques (using the homogenized model) [17-19] or the model order reduction approaches (NTFA) [29]. 


\section{Algorithm 3 Hybrid $\mathrm{FE}^{2}$ algorithm for nonlinear quasi-static} problem

\section{for $k=1$ until convergence do}

- Solve the global equilibrium equation (4) in $\Omega_{M}$

- In the region of interest $\hat{\Omega}_{M}$ at each integration point: use the $\mathrm{FE}^{2}$ model

- Global-to-local information transfer: localization step. Impose the boundary conditions on the local mesh boundaries $\partial \Omega_{m}$ (cf. Fig. 3(b)) derived from the global homogeneous strain (HS) tensor:

HSBC (homogeneous strain boundary conditions): imposed displacement for general REV (with $\boldsymbol{x}_{m}$ being a point on $\partial \boldsymbol{\Omega}_{m}$ ):

$$
\boldsymbol{u}_{m}^{k}\left(\boldsymbol{x}_{m}\right)=\boldsymbol{\varepsilon}_{M}^{k} \boldsymbol{x}_{m} \quad \text { on } \partial \boldsymbol{\Omega}_{m}
$$

or PBC (periodicity boundary condition) for periodic REV (with $\boldsymbol{w}_{m}^{k}$ being a periodic displacement on $\partial \Omega_{m}$ ):

$$
\boldsymbol{u}_{m}^{k}\left(\boldsymbol{x}_{m}\right)=\boldsymbol{\varepsilon}_{M}^{k} \boldsymbol{x}_{m}+\boldsymbol{w}_{m}^{k}\left(\boldsymbol{x}_{m}\right) \quad \text { on } \partial \boldsymbol{\Omega}_{m}
$$

○ Local problems: nonlinear problems full resolution on $\Omega_{m}$ with Algorithm 1 using the local heterogeneous constitutive model

- Local-to-global information transfer: homogenization step. Compute the global stress tensor at the considered integration point by averaging the computed local stress $\boldsymbol{\sigma}_{m}^{k}$ over the REV $\Omega_{m}$ :

$$
\tilde{\boldsymbol{\sigma}}_{M}^{k}=\left\langle\boldsymbol{\sigma}_{m}^{k}\right\rangle_{\Omega_{m}}=\frac{1}{\left|\mu\left(\Omega_{m}\right)\right|} \int_{\Omega_{m}} \boldsymbol{\sigma}_{m}^{k} d \Omega_{m}
$$

with $\mu\left(\Omega_{m}\right)$ the measure of the REV

- In $\Omega_{M} \backslash \hat{\Omega}_{M}$ : integration of the global constitutive law (Eq. (3)) to find $\sigma_{M}^{k}$ - Update the global residual:

$$
\left[R_{M}^{k}\right]= \begin{cases}{\left[F_{M}^{e x t}\right]-\left[F_{M}^{i n t, k}\left(\tilde{\boldsymbol{\sigma}}_{M}^{k}\right)\right]} & \text { in } \hat{\Omega}_{M} \\ {\left[F_{M}^{e x t}\right]-\left[F_{M}^{i n t, k}\left(\boldsymbol{\sigma}_{M}^{k}\right)\right]} & \text { in } \Omega_{M} \backslash \hat{\Omega}_{M}\end{cases}
$$

- Check the convergence on $\left[R_{M}^{k}\right]$

end

It has to be noticed that in the region of interest $\hat{\Omega}_{M}$, the computation of the global stress $\boldsymbol{\sigma}_{M}^{k}$ is avoided. It is substituted with the averaged local stress $\tilde{\boldsymbol{\sigma}}_{M}^{k}$ in the expression of the internal forces $\left[F_{M}^{i n t, k}\left(\tilde{\boldsymbol{\sigma}}_{M}^{k}\right)\right]$. Therefore, the $\mathrm{FE}^{2}$ algorithm aims to minimize the difference between the external forces and internal forces involving corrected stress.

\section{Comparison of the Hybrid $\mathrm{FE}^{2}$ and Local Defect Correction Methods}

$\mathrm{FE}^{2}$ and LDC methods both aim to solve a problem on several levels with a complete global level representing the structure's behavior and finer local levels added to depict more precisely local phenomena. Both computational schemes exploit an iterative multilevel resolution aiming to benefit from local problems resolution to correct on-the-fly the global problem. The multiscale coupling algorithm is carried out in a similar way and can be generalized by Algorithm 4.

Algorithm 4 Unified multiscale coupling algorithm for nonlinear quasi-static problem

\section{for $k=1$ until convergence do}

- Solve the equilibrium equation (4)

- In the region of interest: use local model

- Global-to-local information transfer. Impose the Dirichlet-like boundary conditions on the local mesh boundaries $\partial \Omega_{m}$ derived from the global problem's solution (displacement or associated strain);

○ Local problem(s): nonlinear problem(s) full resolution on $\Omega_{m}$ with Algorithm 1 (can be done with any discretization method);

o Local-to-global information transfer.
- Integration of the global constitutive law (Eq. (3)) where needed

- Update the global residual:

o based on the fine scale computations in the region of interest;

o via the standard procedure elsewhere (see Algorithm 1)

- Check the convergence.

end

Such multilevel resolution algorithm can be seen as a unified formalism which could be used to interpret a wide range of multiscale coupling methods (e.g., Refs. [7,24]).

The main algorithmic difference between the compared $\mathrm{FE}^{2}$ and LDC approaches is summarized in the information transfer between levels, which is carried out in terms of the primal variable (displacement) for the LDC approach, or the dual variable (strain/stress) for the $\mathrm{FE}^{2}$ technique. It implies a slightly different evaluation in the boundary values and in the global updated residual. For this latter, in the LDC method, the external forces are replaced by an evaluation of the global internal forces associated with the restricted local displacement through the associated global stress (that has only an algorithmic meaning). For the $\mathrm{FE}^{2}$ approach, the internal forces are substituted by the ones derived from the homogenized stress (averaged local stress).

The LDC method can thus be seen as a meso-homogenization approach, as features it aims to represent are localized at the global or intermediate level and therefore local levels generally recover a part of the structure. The main differences between the methods are summarized hereafter:

- LDC method:

o needs the integration of the global constitutive law on the whole structural level;

o does not provide the corrected global stress: necessity to store local levels if one is concerned with local stress state;

$\circ$ in the presence of localized effect, appropriate boundary conditions are imposed to the local problem;

o small-scale separation $\rightarrow$ many levels in order to achieve the microstructural scale.

- $\mathrm{FE}^{2}$ method:

o limited in the presence of localized effects due to the scale separation hypothesis (cf. boundary conditions on REV);

o relevant exclusively in the case of large-scale separation;

o natively represents the microstructure.

As it has been noticed, differences are limited between the two considered classes of multiscale methods. Moreover, a choice sometimes considered in the literature [30] to merge all local $\mathrm{FE}^{2}$ problems in one computation makes the local level of the $\mathrm{FE}^{2}$ method even closer to the one defined with the LDC method. Therefore, it would be interesting to explore the intersection between these two classes of multiscale methods: what is the maximal relevant size of REV and what is the level of details accessible with the LDC approach? Would we obtain the same solutions, and comparable computational costs, if the same level of details could be represented with both methods?

\section{Conclusions}

The present analysis has enabled to place in a unified framework two multiscale methods based on multilevel coupling but developed to handle different types of length scale separation. Being generic, the proposed formalism is not limited to the two compared methods, but can be used to represent several multiscale coupling methods. It allows to clearly reveal strong conceptual and algorithmic similarities. Hence, the locally adaptive multigrid approach can be seen as a meso-homogenization method working on the primal variable (instead of the dual variable for classical homogenization techniques), suitable when the large scale separation argument is no more valid. 


\section{Acknowledgment}

This work was developed within the framework of the MISTRAL joint research laboratory between Aix-Marseille University, CNRS, Centrale Marseille and CEA (Commissariat à l'Énergie Atomique et aux Énergies Alternatives). The authors are grateful to the PLEIADES project, financially supported by the CEA, EDF (Électricité de France) and FRAMATOME, that funded this research work.

\section{Conflict of Interest}

There are no conflicts of interest.

\section{Data Availability Statement}

The datasets generated and supporting the findings of this article are obtainable from the corresponding author upon reasonable request. The authors attest that all data for this study are included in the paper. No data, models, or codes were generated or used for this paper.

\section{References}

[1] E, W., 2011, Principles of Multiscale Modelling, Cambridge University Press, Cambridge, UK

[2] Babuška, I., and Guo, B., 1992, "The h, p and h-p Version of the Finite Element Method; Basis Theory and Applications," Adv. Eng. Softw., 15(3), pp. 159-174.

[3] Fish, J., 1992, "The S-version of the Finite Element Method," Comput. Struct., 43(3), pp. 539-547.

[4] Brandt, A., 1977, "Multi-Level Adaptive Solutions to Boundary-Value Problems," Math. Comput., 31(138), pp. 333-390.

[5] Sanchez-Palencia, E., 1980, Homogenization Method for the Study of Composite Media, Springer, Berlin.

[6] Bornert, M., Bretheau, T., and Gilormini, P., 2001, Homogénéisation En Mécanique Des Matériaux, Tome 1: Matériaux Aléatoires élastiques Et Milieux Périodiques, Hermes Science, Paris.

[7] E, W., and Engquist, B., 2003, "The Heterogeneous Multiscale Methods," Commun. Math. Sci., 1(1), pp. 87-132.

[8] Feyel, F., and Chaboche, J.-L., 2000, "FE² Multiscale Approach for Modelling the Elastoviscoplastic Behaviour of Long Fibre SiC/Ti Composite Materials," Comput. Methods Appl. Mech. Eng., 183(3-4), pp. 309-330.

[9] Dvorak, G. J., 1992, "Transformation Field Analysis of Inelastic Composite Materials," Proc.: Math. Phys. Sci., 437(1900), pp. 311-327.

[10] McCormick, S., 1984, Fast Adaptive Composite Grid (FAC) Methods: Theory for the Variational Case, Springer, Vienna, pp. 115-121.

[11] Hackbusch, W., 1984, Local Defect Correction Method and Domain Decomposition Techniques (Defect Correction Methods: Theory and Applications), Springer, Vienna, pp. 89-113.
[12] Koliesnikova, D., Ramière, I., and Lebon, F., 2020, “A Unified Framework for the Computational Comparison of Adaptive Mesh Refinement Strategies for All-Quadrilateral and All-Hexahedral Meshes: Locally Adaptive Multigrid Methods Versus h-Adaptive Methods," submitted

[13] Barbié, L., Ramière, I., and Lebon, F., 2014, "Strategies Around the Local Defect Correction Multi-Level Refinement Method for Three-Dimensional Linear Elastic Problems," Comput. Struct., 130, pp. 73-90.

[14] Guedes, J., and Kikuchi, N., 1990, "Preprocessing and Postprocessing for Materials Based on the Homogenization Method With Adaptive Finite Element Methods," Comput. Methods Appl. Mech. Eng., 83(2), pp. 143-198.

[15] Hashin, Z., and Shtrikman, S., 1963, "A Variational Approach to the Theory of the Elastic Behaviour of Multiphase Materials," J. Mech. Phys. Solids, 11(2), pp. $127-140$.

[16] Mori, T., and Tanaka, K., 1973, "Average Stress in Matrix and Average Elastic Energy of Materials With Misfitting Inclusions," Acta Metall., 21(5), pp. 571-574.

[17] Neuss, N., Jäger, W., and Wittum, G., 2001, "Homogenization and Multigrid," Computing, 66(1), pp. 1-26.

[18] Gu, H., Réthore, J., Baietto, M.-C., Sainsot, P., Lecomte-Grosbras, P., Venner, C., and Lubrecht, A., 2016, "An Efficient Multigrid Solver for the 3D Simulation of Composite Materials," Comput. Mater. Sci., 112(A), pp. 230-237.

[19] Miehe, C., and Bayreuther, C. G., 2007, "On Multiscale FE Analyses of Heterogeneous Structures: From Homogenization to Multigrid Solvers," Int. J. Numer. Methods Eng., 71(10), pp. 1135-1180.

[20] Brandt, A., 1994, "Rigorous Quantitative Analysis of Multigrid I: Constant Coefficients Two-Level Cycle With $\mathrm{L}_{2}$-Norm," SIAM J. Numer. Anal., 31(6), pp. $1695-1730$

[21] Whitcomb, J., and Woo, K., 1993, "Application of Iterative Global/Local Finite-Element Analysis. Part 2: Geometrically Non-Linear Analysis," Commun. Numer. Methods Eng., 9(9), pp. 757-766.

[22] Gendre, L., Allix, O., Gosselet, P., and Comte, F., 2009, "Non-Intrusive and Exact Global/Local Techniques for Structural Problems With Local Plasticity," Comput. Mech., 44, pp. 233-245.

[23] Passieux, J.-C., Réthoré, J., Gravouil, A., and Baietto, M.-C., 2013, "Local/Global Non-Intrusive Crack Propagation Simulation Using a Multigrid X-FEM Solver," Comput. Mech., 52(6), pp. 1381-1393.

[24] Fish, J., and Belsky, V., 1995, "Multigrid Method for Periodic Heterogeneous Media. Part 2: Multiscale Modeling and Quality Control in Multidimensional Case," Comput. Methods Appl. Mech. Eng., 126(1-2), pp. 17-38.

[25] Barbié, L. Ramière, I, and Lebon, F., 2015, "An Automatic Multilevel Refinement Technique Based on Nested Local Meshes for Nonlinear Mechanics," Comput. Struct., 147, pp. 14-25.

[26] Feyel, F., 1999, "Multiscale FE ${ }^{2}$ Elastoviscoplastic Analysis of Composite Structures," Comput. Mater. Sci., 16(1-2), pp. 344-354.

[27] Miehe, C., Schotte, J., and Schröder, J., 1999, "Computational Micro-Macro Transitions and Overall Moduli in the Analysis of Polycrystals at Large Strains," Comput. Mater. Sci., 16(1-4), pp. 372-382.

[28] Ramière, I., Masson, R., Michel, B., and Bernaud, S., 2017, "Un schéma de calcul multi-échelles de type Éléments Finis au carré pour la simulation de combustibles nucléaires hétérogènes," 13e colloque national en calcul des structures, no. 126623, Université Paris-Saclay, May 2017, Giens, Var, France.

[29] Michel, J., and Suquet, P., 2003, "Nonuniform Transformation Field Analysis," Int. J. Solids Struct., 40(25), pp. 6937-6955.

[30] Peyre, G., 2015, "FE ${ }^{2}$ Method and Hyperreduction: Towards Intensive Computations at the Micro Scale," Ph.D thesis, Ecole Nationale Supérieure des Mines de Paris. 